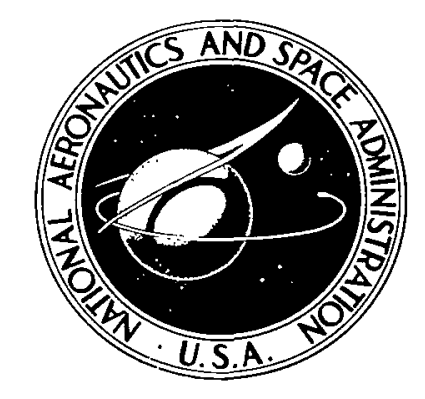

NASA IN D-5611 C.I

LOAN COPY: RETURP

AFWL (DOGL) KIRTLAND AFB, N.

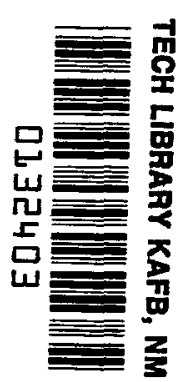

\title{
A PASSIVE METHOD FOR SCANNING THE CELESTIAL SPHERE
}

by Albert J. Fleig, Jr.

Goddard Space Flight Center

Greenbelt, Md. 20771 


\section{Report No. NASA TN D-5611 \\ 4. Title and Subtitle}

A Passive Method for Scanning the Celestial Sphere

7. Author(s)

Albert J. Fleig, Jr.

9. Performing Organization Name and Address

Goddard Space Flight Center

Greenbelt, Maryland 20771

12. Sponsoring Agency Name and Address

National Aeronautics and Space Administration

Washington, D. C. 20546

15. Supplementary Notes

16. Abstract

A passive method for scanning the celestial sphere which is applicable to missions requiring long lifetime and moderate pointing accuracies is described herein. The spacecraft is spin stablilzed and it is the spin axis which is caused to follow the scan pattern. The scan is obtained by combining the gravity gradient induced precession of the spacecraft's spin axis about its orbit pole with the oblateness induced precession of the orbit pole. The parameters directly affecting a particular mission are the orbit altitude, inclination, and eccentricity and the body spin rate, cone angle and inertia difference. Design curves which can be used for parameter trade-off studies of these factors are included.

17. Key Words Suggested by Author Passive scan

Gravity gradient

Spin stabilization

Orbiting radiotelescope

19. Security Classif. (of this report) Unclassified
18. Distribution Statement

Unclassified--Unlimited

20. Security Clossif. (of this poge) Unclassified
21. No. of Pages

5
22. Price* $\$ 3.00$

\footnotetext{
"For sale by the National Technical Information Service, Springfield, Virginia 22151
} 


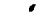

.

4 
CONTENTS

Abstract.................... ii

INTRODUCTION .................. 1

NOTATION ............................... 2

EQUATIONS AND ASSUMPTIONS.......... 3

ADVANTAGES AND PROBLEMS .......... 5

CONCLUSION ................. 5 


\title{
A PASSIVE METHOD FOR SCANNING THE CELESTIAL SPHERE
}

\author{
by \\ Albert J. Fleig, Jr. \\ Goddard Space Flight Center
}

\section{INTRODUCTION}

It is possible to combine two well known perturbations of the motion of a spacecraft to provide a desirable attitude control scheme. The external torque resulting from the gradient of the gravitational field will cause the spin axis of the spacecraft to precess about its orbit pole. The oblateness of the earth causes the spacecraft's orbit to precess about the celestial pole. A judicious combination of these two effects causes the spin axis of the spacecraft to follow a scan pattern as shown in Figure 1. This figure shows the scan pattern for a spacecraft with an orbit inclination of 45 degrees and an angle between the spin axis and the orbit pole of $45 \mathrm{de}-$ grees. If the inclination and cone angle were both 30 degrees, the scan would cover the area from 30 degrees north latitude to the north pole; for an inclination of 85 degrees and a cone angle of 75 degrees it would cover the area from 70 degrees south latitude to 80 degrees north latitude. Other combinations are obviously possible. An inclination of 90 degrees

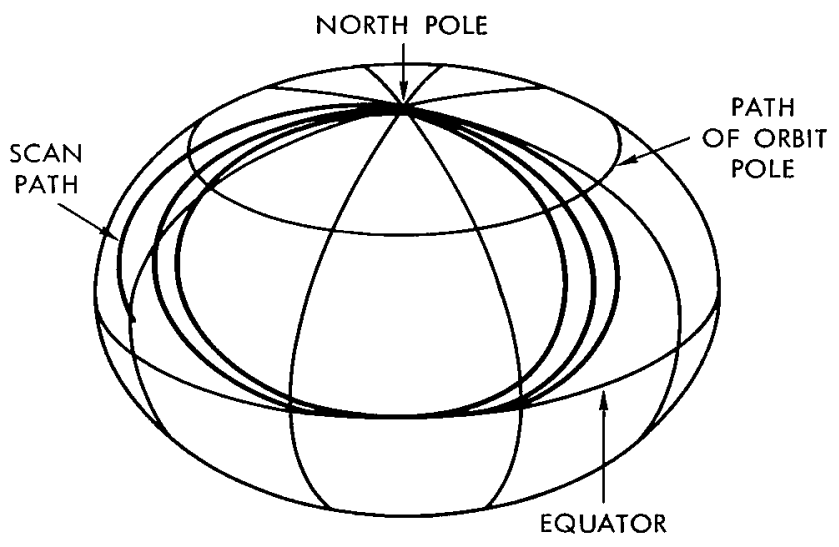

Figure 1-Typical scan pattern. and a cone angle of 90 degrees would cover the entire celestial sphere. Unfortunately an orbit with a 90 degrees inclination does not precess and the gravity gradient torque is zero for a 90 degree cone angle. Thus full coverage from a single combination of conditions is not possible.

One possible mode for obtaining full coverage is to initialize the spacecraft with an inclination of 45 degrees and a cone angle of 45 degrees and scan the northern hemisphere. The spacecraft would then be reinitialized with a cone angle of 135 degrees and would then scan the southern hemisphere. The same active control system which would be used for initial acquisition, and which after a hemisphere scan is used again for reinitialization, could also be activated for brief periods of precision pointing during the nominally passive scan mode. Thus points of particular interest could be accurately studied as they come up in the normal scan mode. In this connection note that each point will be scanned twice in the normal passive mode. 


\section{NOTATION}

Five right-handed orthogonal coordinate frames are defined as a triad of unit vectors for use in this discussion. Each is related to a previously defined frame by one or more rotations specified as ${ }^{i} A(j)$ meaning a rotation about the $i^{\text {th }}$ axis thru an angle $j$. The coordinate frames are:

An inertial frame:

$$
(\underline{X})=\underline{x}_{1}, \underline{x}_{2}, \underline{X}_{3},
$$

where,

$$
\begin{aligned}
& \underline{x}_{1}=\text { first point of Aries, } \\
& \underline{x}_{3}=\text { north celestial pole, and } \\
& \underline{x}_{2}=\underline{x}_{3} \times \underline{x}_{1} .
\end{aligned}
$$

An orbital frame:

$$
(\underline{E})={ }^{3} \mathrm{~A}(\omega){ }^{1} \mathrm{~A}(\mathrm{i}){ }^{3} \mathrm{~A}(\Omega)(\underline{\mathrm{X}}) \text {, }
$$

where,

$$
\begin{aligned}
\omega & =\text { argument of perigee, } \\
\mathrm{i} & =\text { inclination, } \\
\Omega & =\text { longitude of ascending node, } \\
\underline{E}_{1} & =\text { perigee, } \\
\underline{E}_{3} & =\text { orbit pole, and } \\
\underline{E}_{2} & =\underline{E}_{3} \times \underline{E}_{1} .
\end{aligned}
$$

A local vertical frame

$$
(\underline{E})={ }^{3} \mathrm{~A}(\mathrm{v})(\underline{E}) \text {, }
$$

where,

$v=$ true anomaly.

A principal body axis frame

$$
(\underline{e})={ }^{1} \mathrm{~A}(\psi){ }^{2} \mathrm{~A}(\theta){ }^{3} \mathrm{~A}(\phi)\left({ }^{\prime} \underline{E}\right) \text {, }
$$

where,

$$
\begin{aligned}
\underline{e}_{1}= & \text { principal body axis of greatest inertia (spin axis), and } \underline{e}_{2}, \underline{e}_{3} \text { are transverse principal } \\
& \text { axis, } I_{1}>I_{2}=I_{3}
\end{aligned}
$$


A "despun" body frame

$$
\left({ }^{\prime} \underline{e}\right)={ }^{2} \mathrm{~A}(\theta){ }^{3} \mathrm{~A}(\phi)\left({ }^{\prime} \underline{E}\right) \text {. }
$$

\section{EQUATIONS AND ASSUMPTIONS}

The satellite orbit's first-order perturbations resulting from oblateness are wellknown. In particular, the semimajor axis, inclination, and eccentricity have no secular perturbations. The longitude of the node, $\Omega$, argument of perigee, $\omega$, and mean anomaly all have long period variations. In particular, the variation of $\Omega$ can be obtained from

$$
\frac{d \Omega}{d t}=-\frac{3 n}{2}\left(\frac{r_{e}}{a}\right)^{2} \frac{J_{2}}{\left(1-e^{2}\right)^{2}} \cos i
$$

where $\mathrm{J}_{2}=$ oblateness coefficient $=1.08 \times 10^{-3}$; $\mathrm{n}^{2}=\mu / \mathrm{a}^{3}$ where $\mu \approx 398601.0 \mathrm{~km} 3 / \mathrm{sec}^{2} ; \mathrm{a}$ is the semimajor axis in $\mathrm{km}$; $\mathrm{r}_{\mathrm{e}}$, the radius of the earth, is approximately $6378 \mathrm{~km}$; and $\Omega$ is in $\mathrm{rad} / \mathrm{sec}$.

The time for a complete scan of one hemisphere can be determined directly from $\dot{\Omega}$ since a complete scan requires $\Omega$ to increase by $2 \pi$; thus $\mathrm{T}=2 \pi / \dot{\Omega}$. This is a limiting feature of this method since even for a low altitude orbit $(500$ km) a single hemisphere scan takes at least 50 days. The scan period is a function of the semimajor axis, increasing with the $7 / 2$ power of a. Thus the period increases quite rapidly for altitudes above about $5000 \mathrm{~km}$ and this is a second limiting feature. The relationship between a and $\dot{\Omega}$ for three values of $i$ is shown in Figure 2(a). It is possible to cover larger portions of the total sphere with a single scan by increasing $\mathrm{i}$, however the time required to complete the scan also increases. The scan period is also a function of the orbital eccentricity as shown in Figure 2(b). Although the

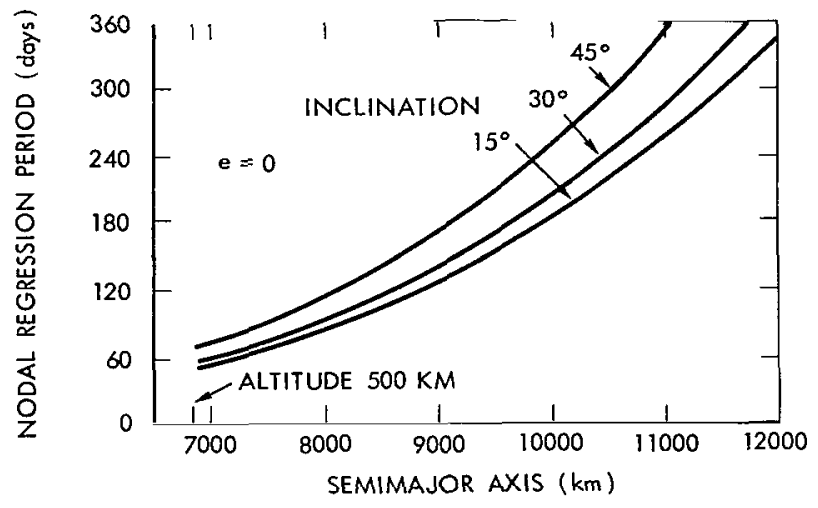

(a)

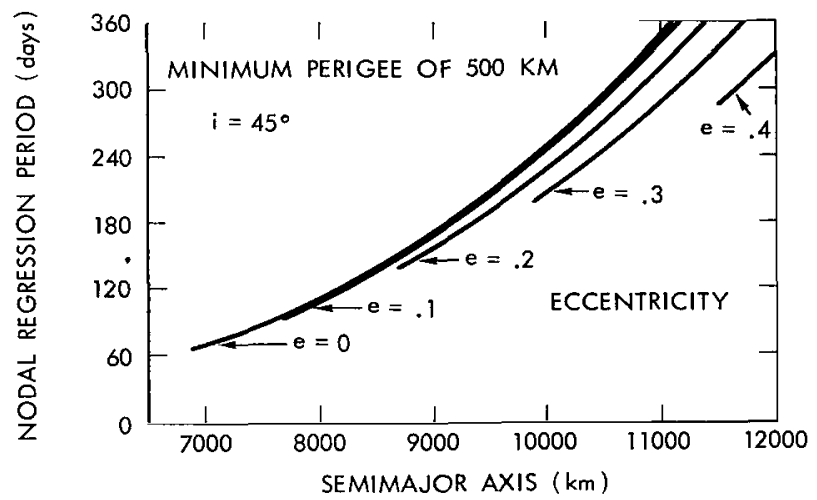

(b)

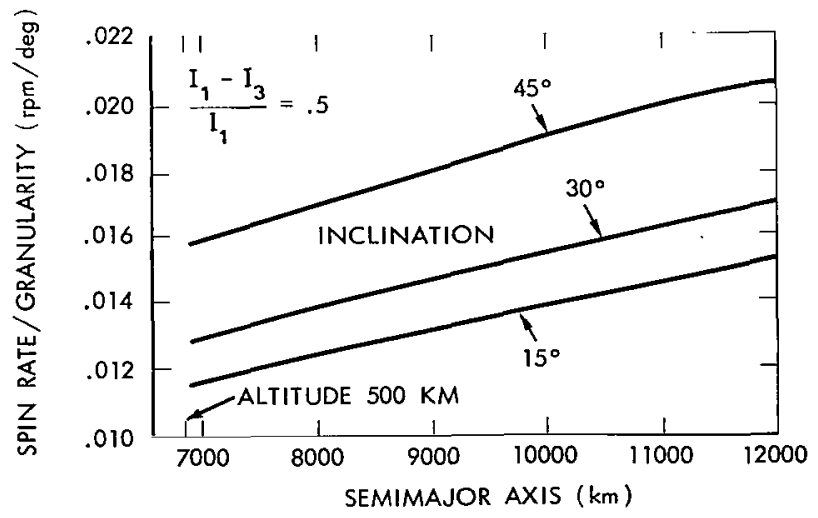

(c)

Figure 2-Trade-off curves showing (a) Effect of inclination, (b) Effect of eccentricity, and (c) Effect of spin rate. 
scan period can be reduced by using an eccentric orbit, there is a drawback to this approach. The gravity gradient induced motion of the spin axis becomes nonuniform, and although much of the or bit can be spent at a higher attitude most of the cone motion will occur at the lowest altitude.

The second part of the scan motion comes from the gravity gradient torque effect on the spacecraft's attitude motion. This torque can be expressed in the local vertical frame as

$$
M=\frac{3 \mu}{r^{3}}\left(I_{1}-I_{3}\right)\left(\sin \theta \cos \theta \cos \phi^{\prime} \underline{E}_{2}+\cos ^{2} \theta \sin \phi \cos \phi^{\prime} \underline{E}_{3}\right)
$$

or in the despun body frame as

$$
M=\frac{3}{2} \frac{\mu}{r^{3}}\left(I_{1}-I_{3}\right)\left(\sin 2 \theta \cos ^{2} \phi^{\prime} \underline{e}_{2}+\cos \theta \sin 2 \phi^{\prime} \underline{e}_{3}\right)
$$

The despun body frame can be related to an inertial frame by the sequence of rotations, $(' \underline{e})={ }^{2} \mathrm{~A}(\theta){ }^{3} \mathrm{~A}(\phi+v+\omega){ }^{1} \mathrm{~A}(\mathrm{i}){ }^{3} \mathrm{~A}(\Omega)(\mathrm{X})$. Under the assumption that the change in body attitude is small over a single orbit, i.e., that $\theta, \phi+v+\omega$, i, and $\Omega$ are constant over a single orbit, and that $\mathrm{v}+\omega \approx \mathrm{nt}$, i.e., for a circular orbit, then $\theta(\mathrm{t})=\theta_{0}$, and $\phi(\mathrm{t})=\phi_{0}-\mathrm{nt}$. Thus the average value over a single orbit of the gravity gradient torque is

$$
\begin{aligned}
M_{a v}=\frac{3 \mu}{2 a^{3}} \frac{n}{2 \pi}\left(I_{1}-I_{3}\right) \times\left[\frac{\sin 2 \theta_{0}}{2} \int_{0}^{2 \pi / n}\left(1+\cos 2\left(\phi_{0}-n t\right)\right) d t^{\prime} \underline{e}_{2}\right. \\
\left.+\cos \theta_{0} \int_{0}^{2 \pi / n} \sin 2\left(\theta_{0}-n t\right) d t^{\prime} \underline{e}_{3}\right]=\frac{3}{4} \frac{\mu}{r^{3}}\left(I_{1}-I_{3}\right) \sin 2 \theta_{0}^{\prime} \underline{e}_{2} .
\end{aligned}
$$

This average torque has an effect similar to that of an offset gyro. The resulting motion is one in which $\theta$ remains constant and $\phi+v+\omega$ increases linearly with time. This is a motion in which the spin axis of the spacecraft precesses about the orbit normal with a constant cone angle and precession rate. The precession rate can be obtained by equating the applied torque to the change in angular momentum

$$
\frac{\mathrm{d}(\phi+\mathrm{v}+\omega)}{\mathrm{dt}}=\frac{3 \mu}{2 \mathrm{r}^{3}} \frac{\left(\mathrm{I}_{1}-\mathrm{I}_{3}\right) \cos \theta}{\mathrm{I}_{1} \dot{\psi}},
$$

where $\dot{\psi}$ is the body spin rate.

There is a relationship between the body cone rate, the orbital regression rate, and the granularity of the scan. For instance, if the angle $\phi+v+\omega$ varies from 0 to $2 \pi$ (i.e., one complete cone motion) while $\Omega$ increases by 3 degrees, then every point in the hemisphere will have passed within $3 / 2$ degree of the control axis after a complete scan pattern is traced. The spin rate required to 
produce a given granularity is shown as a function of the orbit inclination and radius in Figure 2.

There are combinations of cone angle and orbit radius for which the scan pattern is partially occluded by the earth. For some spacecraft the occluding radius may be considered as larger than the earth's radius (e.g., when atmospheric effects on the measur ed quantities are appreciable). The percentage of the total scan pattern which is occluded by various radius spheres is shown in Figure 3.

\section{ADVANTAGES AND PROBLEMS}

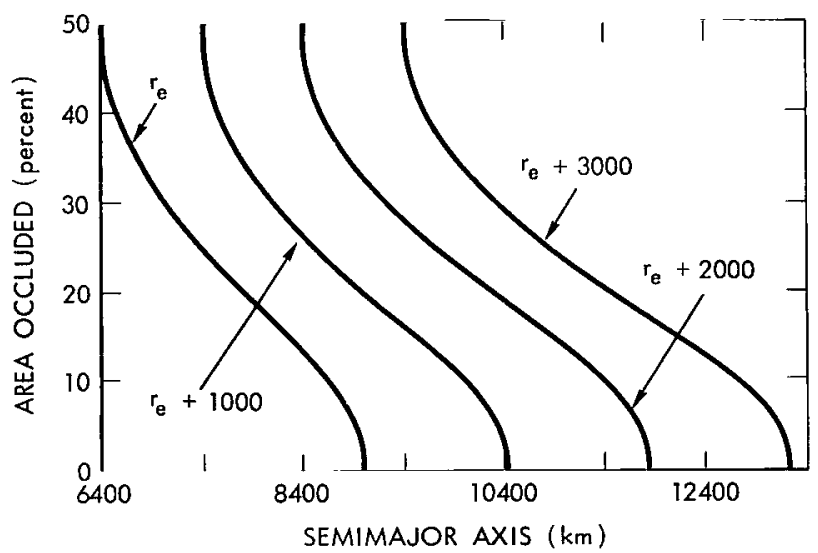

Figure 3-Coverage, for four occluding radii given in kilometers.

There are several problems which must be considered in evaluating this control concept. Although the scan is passive there is a requirement for an active control system for initial alignment, for periods of active pointing, and for reinitialization to start scanning the second hemisphere. The scan pattern discussed here results from a simplified and averaged set of equations. The actual scan is not this uniform and there may be points that do not come within the nominal scan granularity. The scan rate is relatively slow and thus only applicable to long duration experiments. This scheme has the standard advantages of any passive control concept. It is highly reliable, requires a minimum of power, and does not add to the spacecraft weight.

\section{CONCLUSION}

It is possible to select orbit and attitude parameters for a spinning spacecraft so that the spin axis will scan the celestial sphere. The method is applicable to missions of long duration for which a medium altitude orbit and moderate pointing accuracy are acceptable.

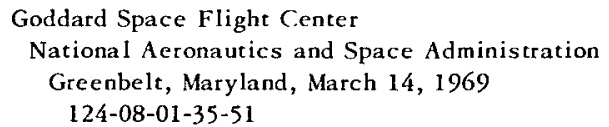




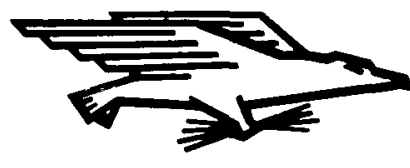

POSTAGE AND FEES PAID NATIONAL AERONAUTICS AND SPACE ADMINISTRATION

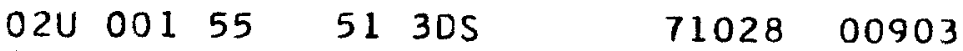 \\ AIR FORCE WEAPONS LABORATORY /WLOL/ \\ KIRTLAND AFB, NEW MEXICD 87117
}

ATT E. LOU BOWMAN, CHIEF, TECH. LIBRARY

POSTMASTER: If Undeliverable (Section 158

"The aeronautical and space activities of the United States shall be conducted so as to contribute. . . to the expansion of buman knowledge of phenomena in the atmosphere and space. The Administration shall provide for the widest practicable and appropriate dissemination of information concerning its activities and the results thereof."

- National Aeronautics and Space ACt of 1958

\title{
NASA SCIENTIFIC AND TECHNICAL PUBLICATIONS
}

TECHNICAL REPORTS: Scientific and technical information considered important, complete, and a lasting contribution to existing knowledge.

TECHNICAL NOTES: Information less broad in scope but nevertheless of importance as a contribution to existing knowledge.

TECHNICAL MEMORANDUMS:

Information receiving limited distribution because of preliminary data, security classification, or other reasons.

CONTRACTOR REPORTS: Scientific and technical information generated under a NASA contract or grant and considered an important contribution to existing knowledge.
TECHNICAL TRANSLATIONS: Information published in a foreign language considered to merit NASA distribution in English.

SPECIAL PUBLICATIONS: Information derived from or of value to NASA activities. Publications include conference proceedings, monographs, data compilations, handbooks, sourcebooks, and special bibliographies.

TECHNOLOGY UTILIZATION PUBLICATIONS: Information on technology used by NASA that may be of particular interest in commercial and other non-aerospace applications. Publications include Tech Briefs, Technology Utilization Reports and Technology Surveys.

Details on the availability of these publications may be obtained from:

SCIENTIFIC AND TECHNICAL INFORMATION OFFICE

NATIONAL AERONAUTICS AND SPACE ADMINISTRATION Washington, D.C. 20546 